\title{
ROLE OF THE HISTOLOGICAL VARIANT FOR THE PROGNOSIS AND COURSE OF THE FOCAL SEGMENTAL GLOMERULOSCLEROSIS
}

\author{
M. Yordanov ${ }^{1}$, J. Ananiev², R. Robeva ${ }^{1}$ \\ ${ }^{1}$ Clinic of Nephrology, Department of Urology and Nephrology, Military Medical Academy - Sofia, Bulgaria \\ ${ }^{2}$ Department of General and Clinical Pathology, Forensic Medicine and Deontology, Faculty of Medicine, \\ Thracian University - Stara Zagora, Bulgaria
}

\begin{abstract}
The focal segmental glomerulosclerosis is characterized by a morphological heterogeneity, most likely reflecting different pathogenetic mechanisms. The Colombian classification distinguishes five morphological types - non-specific (not otherwise specified or classical), perihilar, cellular, a tubular pole (tip) one and a collapsing one. Eighty-one (81) patients were studied. Their distribution according to the histological variant showed the highest frequency of the non-specific (classical) variant $-70.4 \%$, followed by the perihilar variant $-27.20 \%$, the cellular variant $-1.2 \%$ and the collapsing variant $-1.2 \%$. No patients with tip lesions were identified. There were significant differences in the creatinine levels and the glomerular filtration rate (GFR) at the beginning and at the end of the follow-up between patients with the perihilar and the non-specific variants. Patients with the perihilar variant had a better treatment response with a high percentage of patients achieving complete remission - 59.1\%. Patients with the non-specific variant had a high chance of treatment failure $-26.3 \%$ had no effect from treatment. The results of the study give grounds to assume that the histological variant affects the clinical picture, course and therapeutic response in patients with focal segmental glomerulosclerosis. It could be used as a prognostic marker of disease behavior and guide the clinician in treatment decisions.
\end{abstract}

Key words: focal and segmental glomerular sclerosis, histological variants, treatment outcome

Corresponding author: e-mail: maryordmih@abv.bg

RECEIVED: 15 March 2021; ACCEPTED: 5 April 2021

\section{INTRODUCTION}

T he focal segmental glomerulosclerosis is characterized by a morphological heterogeneity, most likely reflecting different pathogenetic mechanisms. The Colombian classification of the focal segmental glomerulosclerosis distinguishes five morphological types as a result of the topography in the glomerulus and the nature of the endocapillary and extracapillary concomitant lesions - non-specific (not otherwise specified or classical), perihilar, cellular, a tubular pole (tip) one and a collapsing one [4, $18,19]$. The observed glomerular damage affects the podocytes or presents as a sclerosing hyalinosis of the glomerulus, causing synechiae between the capillary loops and the Baumann capsule. These lesions affect part of the glomerulus (segmental involvement) and some glomeruli (focal as opposed to diffuse). 
The changes include obstruction of the capillary lumen $[7,12]$. The lesions usually begin with hyalinosis of several capillary loops with a gradual coverage of the capillary glomerulus. The areas of the glomerular basement membrane exposed by the detached podocytes adhere to the parietal sheet of Bauman's capsule and synechiae occur. The process of segmental sclerosis and capillary collapse progresses to sclerosis and obliteration of the entire capillary convolute [16]. The sclerotic glomeruli can undergo a process of "reabsorption" [11].

The five forms of focal segmental glomerulosclerosis have some common characteristics, but also subtype-specific features (Table 1).

The damage to the tubular pole (the tip lesion) affects the part of the glomerular lobe juxtaposed at the tubular pole and includes an adhesion of the Baumann capsule to the apex, a hypercellularity and a presence of foam cells, and/or sclerosis. In contrast, the collapsing variant indicates a segmental or global mesangial consolidation, a capillary glomerular collapse, and an extracapillary epithelial hypertrophy or proliferation. A microcystic tubular dilatation is often observed [15]. The perihilar variant is characterized by the presence of at least one glomerulus with a perihilar hyalinosis with or without sclerosis and over $50 \%$ perihilar lesions in the sclerotic glomeruli. The cellular variant is defined by at least one glomerulus with an endocapillary hypercellularity covering more than $25 \%$ of the glomerular tuft, leading to the obstruction of the capillary lumen. The non-specific variant is determined by excluding all other variants [7].

The clinical response and prognosis could be related to the histological variant. This is best observed for the corticosteroid response to the apical lesions and the aggressive collapse variant [17, 21].

Each histological variant has some effect on the severity of the course and on the prognosis. The top option is considered to be the most favorable in terms of response to the corticosteroid treatment. The perihilar variant also has a relatively better prognosis. The collapse of the lesion is associated with a higher rate of resistance to treatment with corticosteroids and a higher risk of disease progression [5, 6, 12, 19, 21]. So far, there have been only brief observations on the possible relationship between the epidemiologically determined variant and the histological type. Rather, the histological features of the biopsy obtained from each patient allow certain conclusions to be drawn about the prognosis and the possible therapeutic response. More general clinical and morphological correlations that could guide treatment decisions would be of particular interest to the physician.

The aim of the present study was to demonstrate the frequency of the different histological variants in the studied group of patients, as well as the role of the histological variant of focal segmental glomerulosclerosis for the clinical manifestations, the course, the therapeutic response and the prognosis.

\section{MATERIALS AND METHODS}

We studied 81 patients with a focal segmental glomerulosclerosis, biopsied in the period 2008-2020, hospitalized in the Nephrology Clinic of "Sv. Ivan Rilski" hospital until March 2016, and then at the Nephrology Clinic of the Military Medical Academy, Sofia, from 2016 to 2020.

The average age of the patients was $51.1 \pm 14.2$ years, in the range between 18 and 76 years. The puncture kidney biopsy was the main diagnostic method. Biopsy needles with a Moller Medical $\mathrm{GmbH}$, Germany leader were used, with a lumen of $16 \mathrm{G}$ and a length of 15 or $20 \mathrm{~mm}$, depending on the patient's physical characteristics and the depth at which the kidney is located. The procedure was ultrasoundguided biopsy with a Simens Acuson device and a 3.0-6.0 MHz convex transducer.

The histological examination was performed by embedding in paraffin and cut to $2 \mu \mathrm{m}$ thickness. The resulting sections were stained with: hematoxylin eosin, PAS, Masson's trichrome staining method, for the detection of glomerular and interstitial sclerosis;

Table 1. Focal segmental glomerulosclerosis - morphological correlations (D'Agati VD et al. [7])

\begin{tabular}{|l|c|c|c|c|c|c|c|c|}
\hline Variant & Localization & Distribution & Hyalin & Adhesion & PH & MH & GM & AH \\
\hline Collapsing & Anywhere & Segm./Glob. & $-/+$ & $-/+$ & +++ & $-/+$ & $-/+$ & $-/+$ \\
\hline Tip & Apical & Segm. & $+/-$ & $+++/-$ & - & $-/+$ & $-/+$ & $-/+$ \\
\hline Cellular & Anywhere & Segm. & $-/+$ & $-/+$ & - & $-/+$ & $-/+$ & $-/+$ \\
\hline Perihilar & Perichilus & Segm. & $++/-$ & $+++/-$ & - & $-/+$ & $+++/-$ & $++/-$ \\
\hline Non-specific & Anywhere & Segm. & $+/-$ & $++/-$ & $-/+$ & $-/+$ & $+/-$ & $+/-$ \\
\hline
\end{tabular}

Legend: $\mathrm{PH}$ - podocytic hyperplasia; $\mathrm{MH}$ - mesangial hypercellularity; GM - glomerulomegaly; $\mathrm{AH}$ - arteriolohialinosis 
silver impregnation) JMS, to detect changes in GBM and a Congo-red stain, so as to exclude amyloid deposits. The immunofluorescence testing requires $4 \mu \mathrm{m}$ thick cryostat sections that are incubated with fluorochrome anti - human rabbit antisera directed against IgG, IgA, IgM, C1q, C3, C4, fibrinogen, antilambda and anti - kappa.

The renal biopsies were performed in a strict compliance with the generally accepted indications and contraindications for their performance.

The determination of the histological variant was performed according to the Colombian classification.

Treatment with pulse corticosteroids $(8-12 \mathrm{mg} / \mathrm{kg}$ weight) administered intravenously was followed by a conventional heparin 15000-30000E intravenously, with dose adjusted according to the values of aPTT. In the absence of response, cyclophosphamide given in a dose of $1 \mathrm{~g}$ was added monthly for 6 months. The criterion for a complete remission used in the study was a reduction of proteinuria below $0.3 \mathrm{~g} / 24$ hours and a stable creatinine, and for a partial remission - proteinuria from 0.3 to $3.5 \mathrm{~g} / 24 \mathrm{~h}$ with at least $50 \%$ reduction of the urinary protein (compared to the starting levels) and a stable creatinine [7].

Statistical analysis

The data were entered and processed with the statistical package IBM SPSS Statistics 25.0 and MedCalc Version 14.8.1. For a significance level at which the null hypothesis was rejected, $p<0.05$ was assumed.

\section{RESULTS}

The total number of studied patients with focal segmental glomerulosclerosis was 81 . Their distribution according to the histological variant showed the highest frequency of the non-specific (classical) variant $-70.4 \%$, followed by the perihilar variant $27.20 \%$, the cellular one $-1.2 \%$ and the collapsing one $-1.2 \%$. No patients with tip lesions were identified (Fig. 1).

The comparison between the main indicators characterizing the disease process in patients with nonspecific and perihilar variant showed no significant difference in the level of proteinuria at the beginning and end of the follow-up, and statistically significant differences in creatinine and glomerular filtration rate (GFR) at the beginning and end of the followup (Table 2).

The comparison between the proteinuria at the beginning of the follow-up and at the end of it shows a statistically significant decrease, which in the perihilar variant was more pronounced $(p \leq 0.001)$, and less pronounced in the non-specific one $(p \leq$ $0.05)$ - Fig. 2. With regard to creatinine, the perihilar variant showed retention of values with a slight tendency to decrease, while in the group of patients with the non-specific variant creatinine level tended to increase, in both cases without a statistical significance.

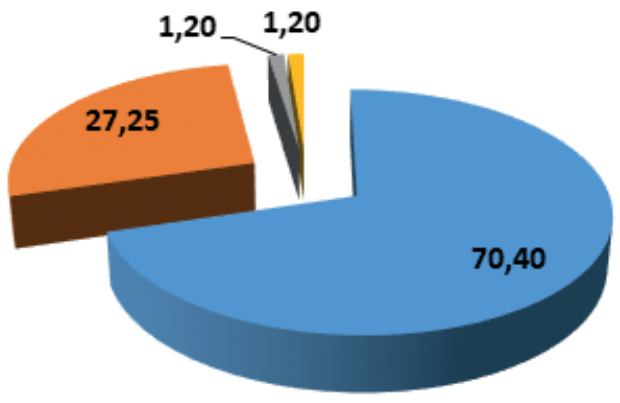

$$
\begin{aligned}
& \text { non-specific variant } \\
& \text { w perihilar variant } \\
& \text { cellular variant } \\
& \text { collapsing variant }
\end{aligned}
$$

Fig. 1. Distribution of the histological variants of focal segmental glomerulosclerosis

Table 2. Main laboratory parameters characterizing the nonspecific and perihilar histological variant

\begin{tabular}{|l|c|c|c|}
\hline Laboratory Indicators & Perihilar variant & Non-specific variant & Statistical Significance \\
\hline Proteinuria at the beginning of the study $(g / 24$ hours) & $2,14 \pm 1,33$ & $3,69 \pm 9,30$ & $n . s$. \\
\hline Proteinuria at the end of the study $(g / 24$ hours) & $0,74 \pm 0,80$ & $1,00 \pm 1,20$ & $n . s$. \\
\hline Creatinine at the beginning of the study $(\mu \mathrm{mol} / \mathrm{l})$ & $115,4 \pm 46,4$ & $155,4 \pm 65,0$ & $\mathrm{p}<0,01$ \\
\hline Creatinine at the end of the study $(\mu \mathrm{mol} / \mathrm{l})$ & $108,6 \pm 45,1$ & $192,4 \pm 136,6$ & $\mathrm{p}<0,001$ \\
\hline Glomerular filtration rate beginning $(\mathrm{ml} / \mathrm{min})$ & $76,27 \pm 29,86$ & $56,68 \pm 25,22$ & $p<0,01$ \\
\hline Glomerular filtration rate ending $(\mathrm{ml} / \mathrm{min})$ & $78,50 \pm 28,99$ & $55,00 \pm 30,76$ & $p<0,01$ \\
\hline
\end{tabular}




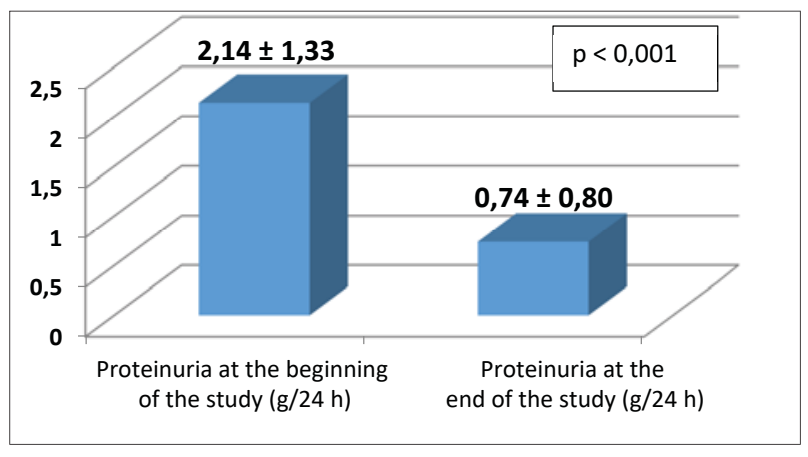

Fig. 2A. Perihilar variant - Proteinuria at the beginning and at the end of the study (g/24 hours)

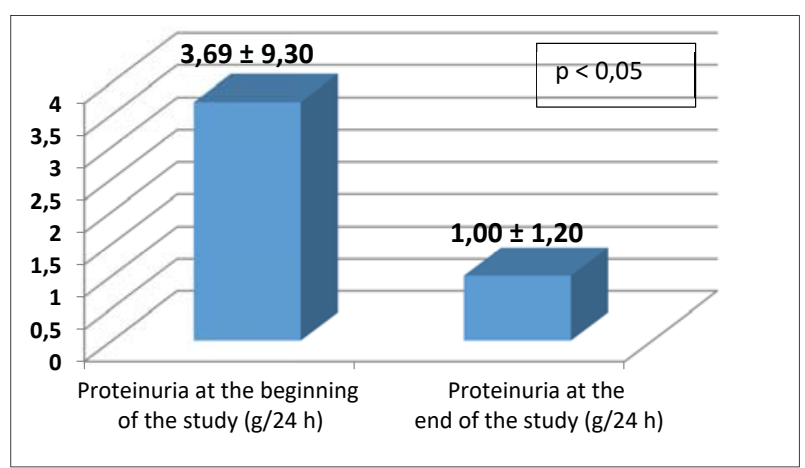

Fig. 2C. Non-specific variant - proteinuria at the beginning and at the end of the study ( $\mathrm{g} / 24$ hours)

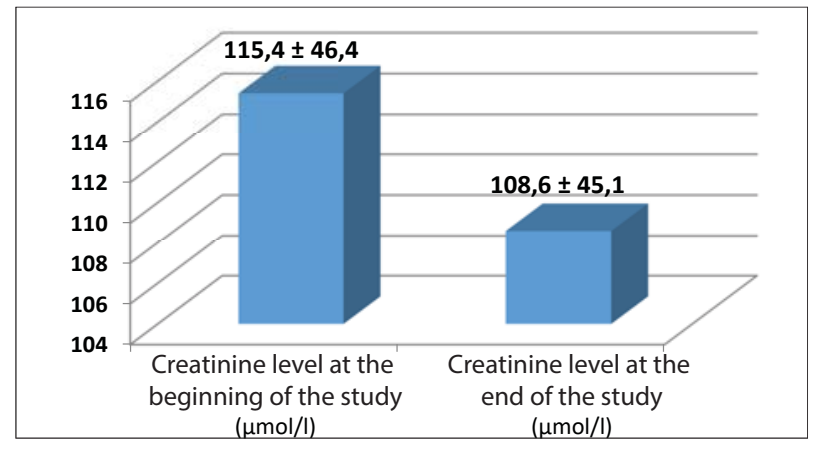

Fig. 2B. Perihilar variant - creatinine level at the beginning and at the end of the study $(\mu \mathrm{mol} / \mathrm{l})$

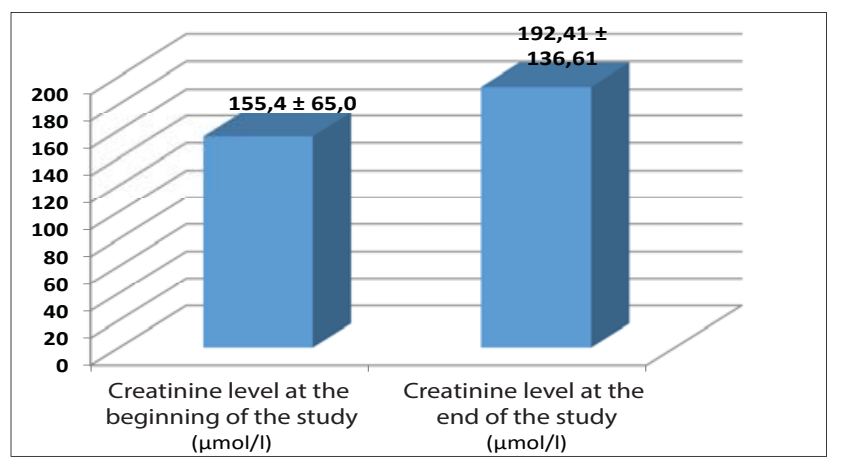

Fig. 2D. Non-specific variant - creatinine level at the beginning and at the end of the study $(\mu \mathrm{mol} / \mathrm{l})$

Fig. 2. (A, B, C, D). Laboratoty indicators at the beginning and at the end of the follow-up

There are differences between the percentage of glomeruli with a global sclerosis, which in the perihilar variant is $13.1 \%$, while in the nonspecific variant it is $27 \%$. In terms of the percentage of coverage by segmental sclerosis, it is $35.1 \%$ for the perihilar variant and $40 \%$ for the nonspecific variant.

Regarding the results of the treatment, there is a statistically significant difference between the patients with a complete remission and those without an effect in the perihilar variant, where a better effect of the therapy was achieved $(p<0.001)$. Treatment response for the perihilar variant was as follows: $9.1 \%$ - without effect; $59.1 \%$ - complete remission; $31,8 \%$ - partial remission.

In the non-specific variant, the differences were not significant. When comparing the percentage of patients who did not respond to treatment, a statistical difference was found with a predominance of the non-specific variant $(p<0.05)$ (treatment response: $26.3 \%$ - without effect; $40.4 \%$ - complete remission; $33.3 \%$ - partial remission).

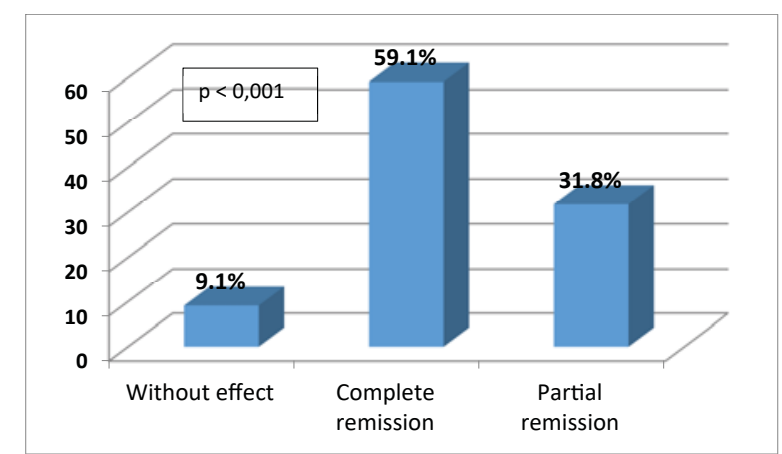

Fig. 3A. Perihilar variant - treatment response: $9.1 \%$ - without effect; $59.1 \%$ - complete remission; 31,8\% - partial remission

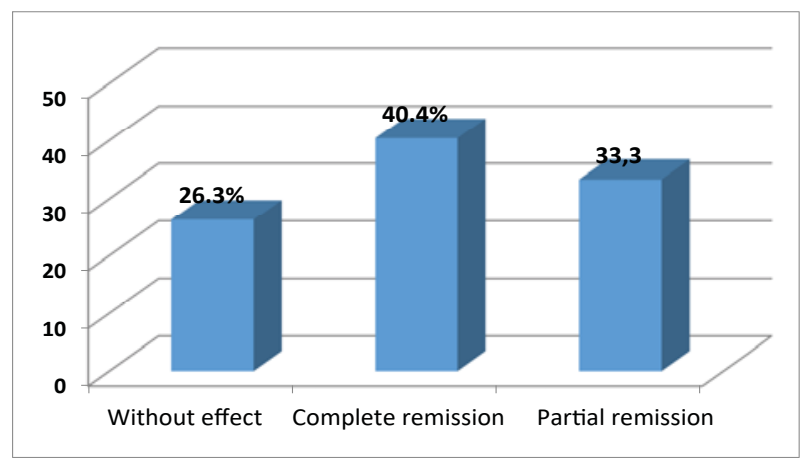

Fig. 3B. Non-specific variant - treatment response: $26.3 \%$ - without effect; $40.4 \%$ - complete remission; 33,3\% - partial remission

Fig. 3 (A, B). Result of the treatment 


\section{DISCUSSION}

Focal segmental glomerulosclerosis is one of the most common causes of primary glomerulonephritis and is the most common finding among renal biopsies [1, 4, 14, 15]; at the same time, it is a leading cause of end-stage renal disease [10]. The disease has a varying and unpredictable clinical course. The Colombian classification systematizes the morphological variants and creates a basis for searching for morphological-clinical and therapeutic interdependencies [7]. The frequency of the different histological variants varies significantly in the different studies. In our study we found that the most common variant was the non-specific variant $-70.4 \%$ of the kidney biopsies, followed by the perihilar one $-27.2 \%$ and the cellular and collapsing variants $-1.2 \%$. It is noteworthy that for a period of 14 years we did not find patients with apical lesions. Our data are similar to those of Thomas et al. [21] with the exception that the non-specific variant takes up the first place with a very low percentage $-42 \%$, followed by the perihilar variant $-26 \%$, and the collapsing one $-11 \%$, while the incidence of the cellular one is $3 \%$. Tip lesions are found in $17 \%$ of the biopsies. Most studies indicate that the nonspecific variant is the most common one, with varying percentages being cited (42-77\%). The incidence of the perihilar variant varies from 4 to $26 \%$, and of tip lesions $-10-17 \%$. The collapsing and especially the cellular variants are rarer $[2,3$, $4,6,9,21]$.

The comparison between the level of proteinuria in patients with non-specific and perihilar variants did not show significant differences, while such were present regarding the level of creatinine and the glomerular filtration rate at the beginning and at the end of the follow-up, which reflects better the baseline renal function and the possibility of a therapeutic response. In the perihilar variant the follow-up established the retention of the values with a tendency for a slight decrease, while in the non-specific variant there was a tendency for a slight increase.

Patients with the perihilar variant responded better to treatment - only $9.1 \%$ did not improve at all after treatment, $59.1 \%$ had a complete remission and $31,8 \%$ - partial remission of the disease.

In contrast to some studies, pointing at hyperfiltration as the main cause of the perihilar variant, we excluded some known causes of secondary kidney disease /severe obesity, hyperfiltration in a single functioning kidney, oligomeganephronia, a low birth weight, urethral reflux, a congenital cyanotic heart disease, sickle cell anemia, diabetes mellitus [2]/. The beneficial effect of the treatment was achieved by the same means used in the non-specific variant. Regarding the non-specific variant, it is assumed that it is a heterogeneous glomerular damage resulting from the intertwining of different pathophysiological mechanisms determining the different effect of therapy [2]. The collapsing variant is severe, often corticoresistant, and usually quickly leads to an end-stage renal disease, as was in our case.

The results of the study give grounds to assume that the histological variant affects the clinical picture, course and therapeutic response in patients with focal segmental glomerulosclerosis. It could be used as a prognostic marker of disease behavior and guide the clinician in treatment decisions.

\section{REFERENCES}

1. Yordanov M, Ananiev Yu, Minkova V, Todorov T. Morphological characteristics of patients with focal and segmental sclerosis. Nephrology, dialysis, transplantation, 2020, 20 (4): 42-49.

2. Arias LF, Jimenez CA, Arroyave MA. Histologic variants of primary focal segmental glomerulosclerosis: presentation and outcome. J Bras Nefrol 2013,35(2):48-53.

3. Arias LF, Henao J, Giraldo RD et al. Glomerular diseases in Hispanic population:review of regional renal biopsy database. Sao Paulo Med J, 2009,127-140.

4. Beaudreuil S, Durrbach A. La hyalinose segmentaire et focale. Bull Acad Natle Med, 2017,201:47-69.

5. Choi J. Histologic classification of FSFS:Does form delineate function. Clin J Am Soc Nephrol 2013,8:344-346.

6. D'Agati V, Alster JM, Jennette JC et al. Association of histologic variants in FSGS clinical trial with presenting features and outcomes. Clin J Am Soc Nephrol, 2013,8(3):399-406.

7. D’Agati VD, Fogo AB, Bruijn JA, Jennette JC. Pathologic classification of focal segmental glomerulosclerosis: A working proposal. Am J Kidney Dis 2004,43:368- 382.

8. Das P, Sharma A, Gupta R et al. Histomorphological classification of focal segmental glomerulosclerosis: a critical evaluation of clinical, histologic and morphometric features. Saudi J Kidney Dis Transpl, 2012,23(5): 1008-1016.

9. Deegens JK, Steenbergan EJ, Borm GF, Wetzels JF. Pathological variants of focal segmental glomerulosclerosisin adult Dutch population- epidemiology and outcome. Nephrol Dial Transplant 2008,23: 186-192.

10. Kitiyakara C, Eggers $\mathrm{P}$, Kopp JB. Twenty-one-year trend in ESRD due to focal segmental glomerulosclerosis in the United Statws. AmJ Kidney Dis, 2004,43:815-825.

11. Kriz W, Le Hir M. Patwhays to nephron loss starting from glomerular disease-insights from animal models. Kidney Int. 2005, 67: 404-419. 
12. Meehan SM, Chang A, Gibson IW et al. A study of interobserver reproducibility of morphologic lesions of focal segmental glomerulosclerosis. Virchows Arch, 2013, 462: 229232.

13. Meliambro K, Schvartzman M, Cravedi P, Campbell KN. The impact of histologic variants of FSGS outcome. Hindawi J, 2014, https://doi.org/10=1155/2014/913690

14. Polito MG, de Moura LA, Kirsztajn GM. An overview on frecancy of renal biopsy diagnosis in Brazil\& clinical and pathological patterns based on 9617 native kidney biopsies. Nephrol Dial Transplant, 2010,25: 490-496.

15. Rosenberg AZ, Kopp JB. Focal Segmental Glomerulosclerosis. Clin J Am Soc Nephrol, 2017, 12:1-16.

16. Schmid H, Henger A, Cohen CD et al. Gene expression profiles of podocyte-associated molecules as diagnostic markers in acquired proteinuric diseases. J Am Soc Nephrol, 2003, 14: 2958-2966.
17. Silverstein DM, Craver R. Presenting features and short-term outcome according to pathologic variant in childhood primary focal segmental glomerulosclerosis. Clin J Am Soc Nephrol, 2007, 2: 700-707.

18. Stokes MB, Markowitz GS, Lan J et al. Glomerular tip lesion:a distinct entity within the minimal change disease/focal segmental glomerulosclerosis spectrum. Kidney Int 2004, 65:1690-1702.

19. Stokes MB, D’Agati VD. Morphologic variants of focal segmental glomerulosclerosis and their significance. Adv Chronic Kidney Dis , 2014, 21: 400-407.

20. Valeri MB, Markowitz AM, D`Agati GS. Cellular focal segmental glomerulosclerosis: Clinical and pathologic features. Kidney Int, 2006,70:1783-1792.

21. Thomas DB, Franceschini N, Hogan SL. Clinical and pathologic characteristics of focal segmental glomerulosclerosis pathologic variants. Kidney Int, 2006, 69: 920-926. 